\title{
Rendimiento hidropónico del asociado de vicia con avena, cebada y trigo en la producción de germinados
}

\author{
Hydroponic yield of the associate of vetch with oats, barley and wheat in the \\ production of germinates
}
Elías Ramos F. ${ }^{1}$, José L. Contreras P. ${ }^{2,4}$, Alfonso G. Cordero F. ${ }^{3}$, James Curasma C. ${ }^{1}$, Briam Jaime Ordoñez M. ${ }^{2}$, M. Esteban Paytan ${ }^{1}$, Yhan Carlos Rojas D.L.C. ${ }^{1}$, Manuel Castrejon V. ${ }^{3}$

\section{Resumen}

El presente trabajo tuvo como objetivo evaluar la variación de la composición química y el rendimiento de las asociaciones avena-vicia, trigo-vicia y cebada-vicia en diversas proporciones de siembra asociada en la forma de cultivos hidropónicos. El estudio se realizó en la ciudad de Huancavelica, Perú, a 3690 msnm. Se emplearon 90 bandejas de 704 $\mathrm{cm}^{2}$, con una densidad de $2.5 \mathrm{~kg}$ de semilla $/ \mathrm{m}^{2}$. Se utilizaron asociados de avena/vicia (A/ $\mathrm{V})$, cebada/vicia (C/V) y trigo/vicia (T/V) con seis proporciones de gramínea/leguminosa: 100/0, 80/20, 60/40, 40/60, 20/80 y 0/100. Se determinó la producción de la biomasa en fresco $(\mathrm{PBF})$ y la composición química de las fracciones porcentuales de materia seca (MS\%), materia orgánica (MO\%), fibra detergente neutra (FDN\%), extracto etéreo (EE\%), proteína cruda (PC\%) y minerales totales (MT\%). Se utilizó un diseño experimental com-

1 Laboratorio de Nutrición Animal y Evaluación de Alimentos, Universidad Nacional de Huancavelica, Perú

2 Escuela de Posgrado-Doctorado de Ciencias Agropecuarias, Universidad Nacional de Huancavelica, Perú

3 Facultad de Ciencias de Ingeniería, Universidad Nacional de Huancavelica, Perú

${ }^{4}$ E-mail: jose.contreras@unh.edu.pe

Recibido: 20 de marzo de 2020

Aceptado para publicación: 16 de julio de 2021

Publicado: 22 de diciembre de 2021

CLos autores. Este artículo es publicado por la Rev Inv Vet Perú de la Facultad de Medicina Veterinaria, Universidad Nacional Mayor de San Marcos. Este es un artículo de acceso abierto, distribuido bajo los términos de la licencia Creative Commons Atribución 4.0 Internacional (CC BY 4.0) [https:// creativecommons.org/licenses/by/4.0/deed.es] que permite el uso, distribución y reproducción en cualquier medio, siempre que la obra original sea debidamente citada de su fuente original 
pletamente al azar, con arreglo factorial de 3x6 (asociado x proporciones) con 5 repeticiones. El asociado, la proporción y la interacción presentaron diferencias significativas $(\mathrm{p}<0.0001)$ sobre el PBF $\left(\mathrm{kg} / \mathrm{m}^{2}\right)$ y la composición química. Los resultados muestran un mayor PBF en C/V (6.79 k/m²), \%MS en A/V (19.58\%), \%FDN en C/V (57.15\%), EE\% en A/ $\mathrm{V}(4.87 \%), \% \mathrm{MM}$ en $\mathrm{C} / \mathrm{V}(4.19 \%)$ y $\% \mathrm{MO}$ en $\mathrm{T} / \mathrm{V}(95.35 \%)$. Las asociaciones $\mathrm{C} / \mathrm{V}$ y A/V presentaron los mejores resultados en PBF y composición química.

Palabras clave: forraje verde hidropónico, hidroforraje, asociado, germinado, hidroponía

\section{AbSTRACT}

The aim of this study was to evaluate the variation of the chemical composition and the performance of the oats-vetch, wheat-vetch and barley-vetch associations in various proportions of sowing in the form of hydroponic crops. The study was carried out in the city of Huancavelica, Peru, at 3690 meters above sea level. In total, 90 trays of $704 \mathrm{~cm}^{2}$ were used, with a density of $2.5 \mathrm{~kg}$ of seed $/ \mathrm{m}^{2}$. Associations of oats $/ \mathrm{vetch}(\mathrm{O} / \mathrm{V})$, barley/ vetch $(B / V)$ and wheat/vetch $(\mathrm{W} / \mathrm{V})$ with six proportions of grass/legume were used: 100 / $0,80 / 20,60 / 40,40 / 60,20 / 80$ and $0 / 100$. The fresh biomass production (FBP) and the chemical composition of the percentage fractions of dry matter (DM\%), organic matter $(\mathrm{OM} \%)$, neutral detergent fibre (NDF\%), ethereal extract (EE\%), crude protein $(\mathrm{CP} \%)$ and total minerals (TM\%) were determined. A completely randomized experimental design was used, with a factorial arrangement of $3 \times 6$ (associations x proportions) with 5 repetitions. The association, the proportion and the interaction showed significant differences $(\mathrm{p}<0.0001)$ on FBP $\left(\mathrm{kg} / \mathrm{m}^{2}\right)$ and chemical composition. The results show a higher FBP in $\mathrm{B} / \mathrm{V}\left(6.79 \mathrm{k} / \mathrm{m}^{2}\right), \% \mathrm{MS}$ in $\mathrm{O} / \mathrm{V}(19.58 \%), \% \mathrm{FDN}$ in $\mathrm{B} / \mathrm{V}(57.15 \%), \mathrm{EE} \%$ in $\mathrm{O} /$ $\mathrm{V}(4.87 \%), \% \mathrm{MM}$ in $\mathrm{B} / \mathrm{V}(4.19 \%)$ and \%MO in $\mathrm{W} / \mathrm{V}(95.35 \%)$. The $\mathrm{B} / \mathrm{V}$ and $\mathrm{O} / \mathrm{V}$ associations presented the best results in FBP and chemical composition.

Key words: hydroponic green forage, hydro-forage, association, sprouts, hydroponics

\section{INTRODUCCIÓN}

La sierra peruana se caracteriza por la siembra de cultivos convencionales y la escasez de pasturas mejoradas. Esta situación se agrava con la disponibilidad reducida del agua de riego, pues se estima una pérdida de los mantos acuíferos, lo que afecta los costos de producción. Un problema similar presenta las aguas almacenadas que no consiguen captar el volumen suficiente, tanto por la baja precipitación y por el uso agrícola inadecuado de dicho recurso (Romero et al., 2009).
En el Perú, los sistemas de producción animal están basados principalmente en la utilización de forrajes. En múltiples casos, a causa de la baja disponibilidad y valor nutricional de las pasturas se tiene un desequilibrio entre los nutrientes consumidos y la demanda fisiológica de animal, lo que limita la productividad en estos sistemas (Villanueva y San Martín, 1997). No obstante, las granjas de explotación pecuaria procuran incrementar la producción y reducir los costos de producción introduciendo insumos alternativos para el mantenimiento de las granjas (Guillermo et al., 2006) Dentro de 
esto, surge como una alternativa la producción de forraje verde hidropónico $(\mathrm{FVH})$, que constituye una tecnología de producción de biomasa adquirida a partir del desarrollo inicial de las plantas en los estados de germinación y crecimiento temprano de plántulas a partir de semillas viables, siendo una siembra de cultivo sin suelo, método que facilita el desarrollo productivo, sostenible y ecológico de las plantas (FAO, 2001; Nuñez y Guerrero 2021). La tecnología de FVH podría satisfacer la creciente demanda de nutrientes para la alimentación del ganado con costos apropiados, además de garantizar una producción constante de cantidad de forraje verde durante todo el año (FAO, 2001).

La producción de forraje hidropónico es una técnica de cultivo que se efectúa en un entorno higiénico libre de químicos y promotores de crecimiento artificial (Jensen y Malter, 1995). El FVH tiene un periodo de crecimiento corto y requiere de una pequeña parcela de tierra para su producción (Mooney, 2005). Es un producto rico en proteínas, fibras, vitaminas y minerales (Chung et al., 1989), con efectos beneficiosos para la salud de los animales (Boue et al., 2003). Por tal razón, el FVH es una de las técnicas agrícolas más importantes que se utilizan actualmente para la producción de forraje verde en muchos países.

Se ha observado que los cultivos asociados de forraje verde hidropónico afectan la calidad nutritiva de los cultivos hidropónicos (Contreras et al., 2015). Por consiguiente, el objetivo de este estudio se centra en evaluar la variación de la composición química y la producción asociada de avena/vicia, trigo/vicia y cebada/vicia en diversas proporciones de siembra como cultivos hidropónicos.

\section{MATERIALes y Métodos}

\section{Lugar de Estudio}

El estudio se llevó a cabo entre junio y setiembre de 2017 en la Universidad Nacio- nal de Huancavelica, Perú. La zona presenta una temperatura promedio diurna de $18.9^{\circ} \mathrm{C}$ y nocturna de $-7{ }^{\circ} \mathrm{C}$, y se encuentra a una altitud de $3690 \mathrm{msnm}$.

\section{Tratamientos}

Se emplearon 90 bandejas de $704 \mathrm{~cm}^{2}$, con una densidad de $2.5 \mathrm{~kg}$ de semilla $/ \mathrm{m}^{2}$, resultando $176 \mathrm{~g}$ de semilla por cada bandeja. Como material biológico se utilizó una leguminosa, vicia (Vicia sativa cv. california) y tres gramíneas, avena (Avena sativa L. cv. Mantaro15), cebada (Hordeum vulgare L. cv. UNA 80) y trigo (Triticum aestivum), con $85 \%$ promedio de germinación en gramíneas (avena cebada y trigo) y $90 \%$ de germinación en leguminosa (vicia). La avena fue adquirida del del Instituto Nacional de Innovación Agraria (INIA-Santa Ana), la vicia de un local comercial de la ciudad de Huancayo y el resto de semillas procedieron de productores de la región Huancavelica, previo pruebas de germinación previo; sin embargo, no se posee información de la pureza varietal. La avena $(\mathrm{A})$, vicia $(\mathrm{V})$, cebada $(\mathrm{C})$ y trigo $(\mathrm{T})$ fueron trabajadas en las siguientes asociaciones: $\mathrm{A} /$ $\mathrm{V}, \mathrm{C} / \mathrm{V}$ y T/V, con seis proporciones de gramínea/leguminosa: 100/0 (176 g / 0 g), 80/20 (140.8 g/35.2 g), 60/40 (105.6 g/ 70.4 g), $40 /$ $60(70.4 \mathrm{~g} / 105.6 \mathrm{~g}), 20 / 80(35.2 \mathrm{~g} / 140.8 \mathrm{~g})$ y $0 / 100(0 \mathrm{~g} / 176 \mathrm{~g})$, con cinco repeticiones por cada proporción/asociaciones, respectivamente. Todos los registros de peso se realizaron con una balanza de precisión $( \pm 0,01 \mathrm{~g})$.

\section{Invernadero}

Se instaló un invernáculo de $3 \mathrm{~m}$ de ancho, $4 \mathrm{~m}$ de largo y $2.5 \mathrm{~m}$ de altura, cubierto con una hoja de polietileno de 0.0078 " de grosor. El invernáculo contenía dos anaqueles metálicos $(1.8 \times 1.0 \mathrm{~m})$ de un nivel para una distribución homogénea de las bandejas.

Las semillas fueron remojadas en una solución de hipoclorito sódico $1 \%$ durante 30 minutos y, posteriormente, lavadas repetidamente en agua fría. Luego, las semillas fueron remojadas durante 24 horas, con dos cam- 
bios de agua. Se utilizó el método indirecto de flotación para las pruebas de germinación (López et al., 2005), a fin de obtener $90 \%$ de germinación en la fase experimental.

Se colocaron las semillas (176 g) en las bandejas para el proceso de germinación, en cantidades por especie de acuerdo con los tratamientos (A/V, C/V y T/V). Las bandejas fueron cubiertas con papel húmedo y plástico negro para evitar el ingreso de luz hasta el día 8, tiempo en que el coleoptilo de las semillas alcanzó $5 \mathrm{~cm}$ promedio de altura. El día 0 fue considerado como el primer día de germinación (colocación de semillas en las bandejas).

Se prepararon dos soluciones nutritivas. Solución A: $340 \mathrm{~g}$ de fosfato de amonio, 2080 $\mathrm{g}$ de nitrato de calcio, $550 \mathrm{~g}$ de nitrato de potasio para $10 \mathrm{~L}$ de agua potable. Solución B: $492 \mathrm{~g}$ de sulfato de magnesio, $0.48 \mathrm{~g}$ de sulfato de cobre, $2.48 \mathrm{~g}$ de sulfato de manganeso, $1.20 \mathrm{~g}$ de sulfato de zinc, $620 \mathrm{~g}$ de ácido bórico, $0.02 \mathrm{~g}$ de molibdato de amonio y $50 \mathrm{~g}$ de quelato de hierro para $4 \mathrm{~L}$ de agua potable.

En los primeros seis días, las bandejas fueron regadas con agua potable por nebulización con ayuda de una mochila pulverizadora de capacidad de $5 \mathrm{~L}$. En el día 7 y 8 se aplicó a cada tratamiento $2.5 \mathrm{ml}$ de la Solución A y $1 \mathrm{ml}$ de la Solución B por cada litro de agua; entre el día 9 y 16 se hizo el riego (solo con agua) por aspersión a las 08:00, 12:00 y 17:00 h. El día 18 se registró del peso del forraje fresco, procediéndose al retiro muestras (bandeja) para su análisis químico. Como muestra se consideró el 100\% de cada tratamiento (bandeja).

\section{Análisis Químico}

Se determinó la producción de la biomasa en fresco (PBF) y la composición química porcentual de la materia seca
(MS\%), materia orgánica (MO\%), fibra detergente neutra (FDN\%), extracto etéreo (EE\%), proteína cruda (PC\%) y minerales totales (MM\%), de acuerdo con las metodologías de la AOAC (1995), utilizando el factor 6.25. Las muestras fueron analizadas en el Laboratorio de Nutrición animal y Evaluación de alimentos - LUNEA, de la Universidad Nacional de Huancavelica, Perú.

\section{Análisis Estadístico}

Se utilizó un diseño completamente al azar, con arreglo factorial de 3 (asociados) $\mathrm{x}$ 6 (proporciones), con cinco repeticiones por tratamiento, de acuerdo con el modelo estadístico Yijk $=\mu+\mathrm{Ci}+\mathrm{Pj}+(\mathrm{AP}) \mathrm{ij}+$ cijk, dónde Yijk $=$ Variable dependiente estudiada; $\mu=$ Media general, $\mathrm{Ai}=$ Efecto del asociado $\mathrm{i}, \mathrm{Pj}$ $=$ Efecto de la proporción $\mathrm{j},(\mathrm{AP}) \mathrm{ij}=$ Efecto de la interacción asociado i con la proporción j; y cijk = Error experimental. Las diferencias entre las medias fueron determinadas mediante la prueba de Tukey con un nivel de probabilidad de 5\%, utilizando el software SAS v. 9.4 .

\section{Resultados y Discusión}

\section{Biomasa en ffFresco (PBF kg/m²)}

El tipo de asociado, la proporción y la interacción de estos indicaron diferencias significativas $(p<0.01)$ sobre el contenido de biomasa en fresco $\left(\mathrm{kg} / \mathrm{m}^{2}\right)$ (Cuadro 1). La producción de biomasa del asociado A/V no tuvo un efecto significativo (Cuadro 2), donde el $6.10 \mathrm{~kg} / \mathrm{m}^{2}$ de PBF en la proporción $100 / 0$ fue reportado similar al $7.00 \mathrm{~kg} / \mathrm{m}$ por Cerrillo et al. (2012). Asimismo, la media de la producción de biomasa fresco de la $\mathrm{A} / \mathrm{V}$ $(100 / 0)$ y C/V $(100 / 0)$ de 6.10 y $8.45 \mathrm{~kg} / \mathrm{m}^{2}$ respectivamente fue inferior a los valores de 16.08 y $15.13 \mathrm{~kg} / \mathrm{m}^{2}$ reportados por Pacco (2018). 
Cuadro 1. Análisis de variancia para determinar el efecto del tipo de asociado avena/vicia, cebada/vicia y trigo/vicia según la proporción de gramínea/leguminosa e interacción asociado/proporción sobre la calidad nutricional del hidropónico

\begin{tabular}{|c|c|c|c|c|c|c|c|c|}
\hline \multirow{2}{*}{$\begin{array}{l}\text { Fuente de } \\
\text { variación }\end{array}$} & \multirow[b]{2}{*}{ GL } & \multicolumn{7}{|c|}{ Cuadrados medios } \\
\hline & & $\begin{array}{c}\text { PBF } \\
\left(\mathrm{kg} / \mathrm{m}^{2}\right)\end{array}$ & $\begin{array}{l}\text { MS } \\
(\%)\end{array}$ & $\begin{array}{l}\mathrm{PC} \\
(\%) \\
\end{array}$ & $\begin{array}{c}\text { FDN } \\
(\%)\end{array}$ & $\begin{array}{l}\mathrm{EE} \\
(\%) \\
\end{array}$ & $\begin{array}{l}\mathrm{MM} \\
(\%)\end{array}$ & $\begin{array}{l}\text { MO } \\
(\%) \\
\end{array}$ \\
\hline A & 2 & $1.49 * *$ & $33.45 * *$ & $53.23 * *$ & $322.2 * *$ & $16.01 * *$ & $0.86^{* *}$ & $0.86^{* *}$ \\
\hline$P$ & 5 & $3.67 * *$ & $22.93 * *$ & $238.0 * *$ & $27.37 * *$ & $5.70 * *$ & $0.74 * *$ & $0.74 * *$ \\
\hline $\mathrm{AxP}$ & 10 & $1.53 * *$ & $18.93 * *$ & $2.51 * *$ & $21.17 * *$ & $2.71 * *$ & $0.27 * *$ & $0.27 * *$ \\
\hline Error & 18 & 0.39 & 0.91 & 1.04 & 8.59 & 0.13 & 0.02 & 0.02 \\
\hline Medias & & 6.12 & 17.69 & 28.19 & 51.17 & 3.65 & 3.92 & 96.07 \\
\hline CV $(\%)$ & & 9.59 & 5.40 & 3.62 & 5.73 & 9.95 & 4.53 & 0.14 \\
\hline
\end{tabular}

$\mathrm{A}=$ Asociado; $\mathrm{P}=$ Proporción; GL: grados de libertad

PBF: biomasa en fresco, MS\%: materia seca; MO\%: materia orgánica; FDN\%: fibra detergente neutra; $\mathrm{EE} \%$ : extracto etéreo, $\mathrm{PC} \%$ : proteína cruda; $\mathrm{MM} \%$ : minerales totales

$* p<0.05 ;{ }^{* *} p<0.01$

Cuadro 2. Efecto de la interacción Proporción x Asociado sobre la producción de biomasa en fresco $\left(\mathrm{kg} / \mathrm{m}^{2}\right)$ de los asociados Avena/Vicia (A/V), Cebada/Vicia (C/V) y Trigo/Vicia (T/V) como cultivos hidropónicos

\begin{tabular}{ccccc}
\hline \multirow{2}{*}{ Proporciones } & \multicolumn{3}{c}{ Asociado } & \multirow{2}{*}{ Promedio } \\
\cline { 2 - 4 } & $\mathrm{A} / \mathrm{V}$ & $\mathrm{C} / \mathrm{V}$ & $\mathrm{T} / \mathrm{V}$ & \\
\hline $100 / 0$ & $6.10^{\mathrm{a}}$ & $8.45^{\mathrm{a}}$ & $8.27^{\mathrm{a}}$ & $7.60^{\mathrm{a}}$ \\
$80 / 20$ & $5.61^{\mathrm{a}}$ & $6.94^{\mathrm{abc}}$ & $6.88^{\mathrm{ab}}$ & $6.47^{\mathrm{abc}}$ \\
$60 / 40$ & $4.97^{\mathrm{a}}$ & $5.65^{\mathrm{c}}$ & $7.13^{\mathrm{ab}}$ & $5.92^{\mathrm{c}}$ \\
$40 / 60$ & $5.29^{\mathrm{a}}$ & $6.19^{\mathrm{bc}}$ & $5.29^{\mathrm{b}}$ & $5.59^{\mathrm{c}}$ \\
$20 / 80$ & $7.11^{\mathrm{a}}$ & $6.07^{\mathrm{bc}}$ & $5.64^{\mathrm{b}}$ & $6.27^{\mathrm{bc}}$ \\
$0 / 100$ & $7.67^{\mathrm{a}}$ & $7.44^{\mathrm{ab}}$ & $6.75^{\mathrm{ab}}$ & $7.29^{\mathrm{ab}}$ \\
\hline Promedio & $6.12^{\mathrm{B}}$ & $6.79^{\mathrm{A}}$ & $6.66^{\mathrm{AB}}$ & \\
\hline
\end{tabular}

$a, b, c$ Medias con diferente letra dentro de columnas son significativamente diferentes $(p<.0 .05)$

$A, B$ Medias con diferente letra dentro de filas son significativamente diferentes $(p<.0 .05)$ 
La producción de biomasa de los asociados $\mathrm{C} / \mathrm{V}$ y $\mathrm{T} / \mathrm{V}$, dados por las proporciones de $100 / 0\left(8.45\right.$ y $8.27 \mathrm{~kg} / \mathrm{m}^{2}$, respectivamente) fueron mayores, para luego ir disminuyendo y aumentando regularmente hasta llegar a la proporción $0 / 100$, cuyas producciones fueron de 7.44 y $6.75 \mathrm{~kg} / \mathrm{m}^{2}$, respectivamente (Cuadro 2). En el caso de la cebada, Contreras et al. (2015) reportaron 9.36 $\mathrm{kg} / \mathrm{m}^{2}$, siendo similar a lo reportado en este estudio $\left(8.45 \mathrm{~kg} / \mathrm{m}^{2}\right)$, pero inferior al valor de $18.14 \mathrm{~kg} / \mathrm{m}^{2}$ reportado por Ghazi et al. (2012) para la proporción 100/0. Para trigo, Cerrillo et al. (2012) y Ghazi et al. (2012) reportaron 7.0 y $11.9 \% \mathrm{~kg} / \mathrm{m}^{2}$ de PBF, respectivamente, mientras que Sánchez et al. (2013) obtuvieron valores entre 30.88 y $30.23 \mathrm{~kg} / \mathrm{m}^{2}$ para diferentes densidades de siembra bajo condiciones de invernadero, valores muy superiores a los $8.27 \mathrm{~kg} / \mathrm{m}^{2}$ reportado en este estudio, diferencias que pueden ser atribuidas al tiempo de cosecha y densidad de siembra.

\section{Materia Seca (\%MS)}

$\mathrm{El}$ asociado $\mathrm{A} / \mathrm{V}$ en las proporciones 100/0, 80/20, 60/40 y 40/60 (Cuadro 3) fueron estadísticamente similares en el tenor de MS (19.80, 23.24, 23.5 y 20.14\%, respectivamente) y estadísticamente superiores al resto de proporciones. En forma similar, se observan valores mayores de producción de MS en la asociación C/V en las proporciones $60 / 40$ y $20 / 80$ y en las proporciones $40 / 60$, $20 / 80$ y $0 / 100 \mathrm{del}$ asociado $\mathrm{T} / \mathrm{V}$.

El tenor de materia seca de 14.07 y $14.11 \%$ encontrado para el cultivo hidropónico cebada/vicia (proporción $0 / 100$ y $0 / 100$, respectivamente) está en línea con los valores obtenidos por Contreras et al. (2015), pero es inferior al 19.26\% reportado por Fazaeli et al. (2011) y superior a los valores hallados por otros autores (Mazuelos, 1995; Silva, 1994; Castro et al., 1998; Lomeli, 2000). De otra parte, el $12-04 \%$ de MS reportado por Lomeli (2000) para el cultivo hidropónico de trigo fue similar al $12.42 \%$ encontrado en el presente estudio para la proporción 100/0, a pesar del menor periodo de crecimiento (12 días) del cultivo hidropónico; en tanto que el $20.1 \%$ de MS reportado por Fuentes et al. (2011) para el cultivo hidropónico de avena fue cercano al valor de $19.80 \%$ (A/V 100/0) del presente estudio. Asimismo, Carrasco y Espinoza (2016) reportaron $19.80 \%$ de MS para la avena.

\section{Proteína Cruda (\%PC)}

Las medias de proteína cruda (Cuadro 4) muestran que el efecto de la proporción se debe exclusivamente al asociado trigo/vicia, donde las proporciones $0 / 100$ y $20 / 80$ presentaron el mayor tenor de PC (38.54 y $35.01 \%$, respectivamente) que la proporción de 100/0 (trigo/vicia) cuyo contenido proteico fue de $24.55 \%$. Por otro lado, dentro de avena/vicia y cebada/vicia, la diferencia entre los valores extremos en el tenor de PC fue 18.31 y $16.63 \%$, respectivamente, mientras que para trigo/vicia fue de $13.99 \%$, lo cual indica que el efecto de la proporción depende del asociado.

Asimismo, dentro de la proporción gramínea/leguminosa (100/0), la diferencia en el contenido de PC fue de $6.65 \%(\mathrm{~T} / \mathrm{V}, \mathrm{A} / \mathrm{V})$ y $4.81 \%(\mathrm{~T} / \mathrm{V}, \mathrm{C} / \mathrm{V})$, mientras que en la proporción $0 / 100$ fue de $2.33 \%(\mathrm{~T} / \mathrm{V}, \mathrm{A} / \mathrm{V}) \mathrm{y}$ $2.17 \%(\mathrm{~T} / \mathrm{V}, \mathrm{C} / \mathrm{V})$ de $\mathrm{PC}$, indicando que el efecto del tipo de asociado depende de la proporción utilizada. Contreras et al. (2015) reportaron medias de las asociaciones de cebada/arveja y trigo/arveja de 20.27 y $24.46 \%$, respectivamente, siendo diferentes del $27.01 \%$ para la cebada/vicia y del $30.62 \%$ para el trigo/vicia, evidenciándose mayor producción de proteína en el presente estudio. El valor de 19.74\% de cebada/vicia (proporción 100/0) es superior al $13.69 \%$ reportado por Fazaeli et al. (2011). El 17.9\% de PC para la avena (A/V 100/0) en esta investigación fue similar al $19.0 \%$ obtenido por Cerrillo et al. (2012) en condiciones similares de siembra, pero superior a 14.48 y $8 \%$ reportados por Fuentes et al (2011) y Carrasco y Espinoza (2016), respectivamente, pudiéndose atribuirse estas diferencias a la pérdida de biomasa (Sneath y Macintosh, 2003). 
Cuadro 3. Efecto de la interacción Proporción x Asociado sobre el contenido de materia seca (\%) de los asociados Avena/Vicia (A/V), Cebada/Vicia (C/V) y Trigo/Vicia (T/V) como cultivos hidropónicos

\begin{tabular}{ccccc}
\hline \multirow{2}{*}{ Proporciones } & \multicolumn{3}{c}{ Asociado } & \multirow{2}{*}{ Promedio } \\
\cline { 2 - 4 } & $\mathrm{A} / \mathrm{V}$ & $\mathrm{C} / \mathrm{V}$ & $\mathrm{T} / \mathrm{V}$ & \\
\hline $100 / 0$ & $19.80^{\mathrm{ab}}$ & $14.07^{\mathrm{c}}$ & $12.42^{\mathrm{c}}$ & $15.43^{\mathrm{b}}$ \\
$80 / 20$ & $23.24^{\mathrm{a}}$ & $16.24^{\mathrm{b}}$ & $15.62^{\mathrm{bc}}$ & $18.37^{\mathrm{a}}$ \\
$60 / 40$ & $23.53^{\mathrm{a}}$ & $18.79^{\mathrm{a}}$ & $15.27^{\mathrm{bc}}$ & $19.19^{\mathrm{a}}$ \\
$40 / 60$ & $20.14^{\mathrm{ab}}$ & $16.86^{\mathrm{b}}$ & $21.58^{\mathrm{a}}$ & $19.47^{\mathrm{a}}$ \\
$20 / 80$ & $17.88^{\mathrm{b}}$ & $18.71^{\mathrm{a}}$ & $19.43^{\mathrm{ab}}$ & $18.67^{\mathrm{a}}$ \\
$0 / 100$ & $12.93^{\mathrm{c}}$ & $14.11^{\mathrm{c}}$ & $18.00^{\mathrm{ab}}$ & $15.01^{\mathrm{b}}$ \\
\hline Promedio & $19.58^{\mathrm{A}}$ & $16.43^{\mathrm{B}}$ & $17.05^{\mathrm{B}}$ & \\
\hline
\end{tabular}

$a, b, c$ Medias con diferente letra dentro de columnas son significativamente diferentes $(p<.0 .05)$

$A, B$ Medias con diferente letra dentro de filas son significativamente diferentes $(p<.0 .05)$

Cuadro 4. Efecto de la interacción Proporción $\mathrm{x}$ Asociado sobre el contenido de proteína cruda (PC\%) de los asociados Avena/Vicia (A/V), Cebada/Vicia $(\mathrm{C} / \mathrm{V})$ y Trigo/Vicia $(\mathrm{T} / \mathrm{V})$ como cultivos hidropónicos

\begin{tabular}{ccccc}
\hline \multirow{2}{*}{ Proporciones } & \multicolumn{3}{c}{ Asociado } & \multirow{2}{*}{ Promedio } \\
\cline { 2 - 4 } & $\mathrm{A} / \mathrm{V}$ & $\mathrm{C} / \mathrm{V}$ & $\mathrm{T} / \mathrm{V}$ & \\
\hline $100 / 0$ & $17.90^{\mathrm{d}}$ & $19.74^{\mathrm{d}}$ & $24.55^{\mathrm{c}}$ & $20.73^{\mathrm{d}}$ \\
$80 / 20$ & $20.41^{\mathrm{d}}$ & $19.55^{\mathrm{d}}$ & $26.71^{\mathrm{bc}}$ & $21.88^{\mathrm{d}}$ \\
$60 / 40$ & $26.77^{\mathrm{c}}$ & $26.20^{\mathrm{c}}$ & $29.95^{\mathrm{b}}$ & $27.65^{\mathrm{c}}$ \\
$40 / 60$ & $27.69^{\mathrm{c}}$ & $28.49^{\mathrm{bc}}$ & $29.95^{\mathrm{b}}$ & $28.70^{\mathrm{c}}$ \\
$20 / 80$ & $32.58^{\mathrm{b}}$ & $31.70^{\mathrm{b}}$ & $35.01^{\mathrm{a}}$ & $33.09^{\mathrm{b}}$ \\
$0 / 100$ & $36.21^{\mathrm{a}}$ & $36.37^{\mathrm{a}}$ & $38.54^{\mathrm{a}}$ & $37.04^{\mathrm{a}}$ \\
\hline Promedio & $26.92^{\mathrm{B}}$ & $27.01^{\mathrm{B}}$ & $30.62^{\mathrm{A}}$ & \\
\hline
\end{tabular}

$a, b, c$ Medias con diferente letra dentro de columnas son significativamente diferentes $(p<.0 .05)$

$A, B$ Medias con diferente letra dentro de filas son significativamente diferentes $(p<.0 .05)$ 
Cuadro 5. Efecto de la interacción Proporción x Asociado sobre el contenido de la Fibra detergente neutra $(\% \mathrm{FDN})$ de los asociados Avena/Vicia (A/V), Cebada/Vicia (C/V) y Trigo/Vicia (T/V) como cultivos hidropónicos

\begin{tabular}{ccccc}
\hline \multirow{2}{*}{ Proporciones } & \multicolumn{3}{c}{ Asociado } & $\begin{array}{c}\text { Media / } \\
\text { Proporción }\end{array}$ \\
\cline { 2 - 4 } & $\mathrm{A} / \mathrm{V}$ & $\mathrm{C} / \mathrm{V}$ & $\mathrm{T} / \mathrm{V}$ & $51.59^{\mathrm{ab}}$ \\
\hline $100 / 0$ & $47.87^{\mathrm{ab}}$ & $56.82^{\mathrm{a}}$ & $50.07^{\mathrm{a}}$ & $49.47^{\mathrm{ab}}$ \\
$60 / 20$ & $42.92^{\mathrm{b}}$ & $58.33^{\mathrm{a}}$ & $47.17^{\mathrm{a}}$ & $48.45^{\mathrm{b}}$ \\
$40 / 40$ & $43.15^{\mathrm{ab}}$ & $55.52^{\mathrm{a}}$ & $46.70^{\mathrm{a}}$ & $50.44^{\mathrm{ab}}$ \\
$20 / 80$ & $53.80^{\mathrm{a}}$ & $52.82^{\mathrm{a}}$ & $44.71^{\mathrm{a}}$ & $52.97^{\mathrm{ab}}$ \\
$0 / 100$ & $50.37^{\mathrm{ab}}$ & $60.09^{\mathrm{a}}$ & $48.47^{\mathrm{a}}$ & $54.10^{\mathrm{a}}$ \\
\hline Media/ consociado & $49.43^{\mathrm{ab}}$ & $59.31^{\mathrm{a}}$ & $53.57^{\mathrm{a}}$ & 51.06 \\
\hline A,B Medias seguidas por letras mayúsculas diferentes en una misma columna difieren entre sí, \\
por la prueba de Tukey a 5\% de probabilidad \\
a,b Medias seguidas por letras minúsculas diferentes en una misma fila difieren entre sí, por \\
la prueba de Tukey a 5\% de probabilidad
\end{tabular}

\section{Fibra Detergente Neutra (\%FDN)}

El análisis de variancia (Cuadro 1) para el efecto del tipo de asociado, proporción e interacción evidenció diferencias estadísticas significativas $(\mathrm{p}<0.01)$; no obstante, los asociados $\mathrm{C} / \mathrm{V}$ y $\mathrm{T} / \mathrm{V}$ no difirieron significativamente entre su proporciones (Cuadro 5). También se observó un mayor y significativo porcentaje de FDN de las medias del asociado cebada/vicia con relación a los otros dos asociados. El porcentaje de FDN para el asociado avena/vicia (proporción 100/0) está en línea con el $49.8 \%$ obtenido por Cerrillo et al. (2012), aunque inferior al 56\% de FDV reportado por Carrasco y Espinoza (2016), evidenciándose que la proporción 100/0 de avena/vicia es menos fibrosa.

\section{Extracto Etéreo (\%EE)}

El análisis de variancia (Cuadro 1) para el efecto del tipo de asociado, proporción e- interacción evidenció diferencias estadísticas significativas $(p<0.0001)$. Las medias (Cuadro 6) muestran que el efecto de la proporción se debe exclusivamente a la mezcla avena/vicia, donde la proporción 100/0 (avena/vicia) presentó el mayor tenor de EE (8.71\%) que la proporción de $0 / 100(2.70 \%)$. El $10.1 \%$ de EE de la avena/vicia (100/0) obtenidos por Cerrillo et al., (2012) fue superior al $8.71 \%$ reportado en el presente estudio, y casi similar al 7.76 y $6.3 \% \%$ obtenidos por Fuentes et al. (2011) en 13 y 16 días de cosecha, respectivamente. Así mismo, el $2.25 \%$ de EE de la mezcla trigo/vicia (100/0) del presente estudio fue similar al 2.0 y $3.18 \%$ de EE reportados por Cerrillo et al. (2012) y Carrasco y Espinoza (2016). El valor obtenido es además similar al 1.0\% de la zona de raíces y $3.8 \%$ de la parte aérea encontrados por Sánchez et al., (2013). De otra parte, el $4.47 \%$ de EE del asociado C/V (100/0) fue similar a los valores obtenidos por Cayllahua et al. (2015) y Carrasco y Espinoza (2016). 
Cuadro 6. Efecto de la interacción Proporción x Asociado sobre el contenido de extracto etéreo (\%EE) de los asociados Avena/Vicia (A/V), Cebada/Vicia $(\mathrm{C} / \mathrm{V})$ y Trigo/Vicia (T/V) como cultivos hidropónicos

\begin{tabular}{ccccc}
\hline \multirow{2}{*}{ Proporciones } & \multicolumn{3}{c}{ Asociado } & $\begin{array}{c}\text { Media / } \\
\text { Proporción }\end{array}$ \\
\cline { 2 - 4 } & $\mathrm{A} / \mathrm{V}$ & $\mathrm{C} / \mathrm{V}$ & $\mathrm{T} / \mathrm{V}$ & $5.14^{\mathrm{a}}$ \\
\hline $100 / 0$ & $8.71^{\mathrm{a}}$ & $4.47^{\mathrm{a}}$ & $2.25^{\mathrm{c}}$ & $4.25^{\mathrm{b}}$ \\
$60 / 20$ & $5.49^{\mathrm{b}}$ & $3.69^{\mathrm{bc}}$ & $3.56^{\mathrm{a}}$ & $3.76^{\mathrm{bc}}$ \\
$60 / 40$ & $4.10^{\mathrm{bc}}$ & $4.04^{\mathrm{ab}}$ & $3.14^{\mathrm{b}}$ & $3.53^{\mathrm{c}}$ \\
$40 / 60$ & $5.10^{\mathrm{bc}}$ & $3.30^{\mathrm{cd}}$ & $2.19^{\mathrm{c}}$ & $2.68^{\mathrm{d}}$ \\
$20 / 80$ & $3.15^{\mathrm{bc}}$ & $2.85^{\mathrm{de}}$ & $2.04^{\mathrm{c}}$ & $2.56^{\mathrm{d}}$ \\
$0 / 100$ & $2.70^{\mathrm{c}}$ & $2.68^{\mathrm{e}}$ & $2.29^{\mathrm{c}}$ & \\
\hline Media/ consociado & $4.87^{\mathrm{A}}$ & $3.50^{\mathrm{B}}$ & $2.58^{\mathrm{C}}$ & \\
\hline A,B & Medias seguidas por letras mayúsculas diferentes en una misma columna difieren entre \\
sí, por la prueba de Tukey a 5\% de probabilidad. & \\
a,b Medias seguidas por letras minúsculas diferentes en una misma fila difieren entre sí, por \\
la prueba de Tukey a 5\% de probabilidad
\end{tabular}

Cuadro 7. Efecto de la interacción Proporción x Asociado sobre el contenido de minerales totales (\%MM) de los asociados Avena/Vicia (A/V), Cebada/Vicia (C/V) y Trigo/Vicia (T/V) como cultivos hidropónicos

\begin{tabular}{ccccc}
\hline \multirow{2}{*}{ Proporciones } & \multicolumn{3}{c}{ Asociado } & $\begin{array}{c}\text { Media / } \\
\text { Proporción }\end{array}$ \\
\cline { 2 - 4 } & $\mathrm{A} / \mathrm{V}$ & $\mathrm{C} / \mathrm{V}$ & $\mathrm{T} / \mathrm{V}$ & $3.57^{\mathrm{b}}$ \\
\hline $100 / 0$ & $3.18^{\mathrm{b}}$ & $4.14^{\mathrm{ab}}$ & $3.38^{\mathrm{c}}$ & $3.71^{\mathrm{b}}$ \\
$60 / 20$ & $3.49^{\mathrm{b}}$ & $4.36^{\mathrm{a}}$ & $3.30^{\mathrm{c}}$ & $3.57^{\mathrm{b}}$ \\
$40 / 40$ & $3.34^{\mathrm{b}}$ & $3.60^{\mathrm{b}}$ & $3.77^{\mathrm{b}}$ & $4.30^{\mathrm{a}}$ \\
$20 / 80$ & $4.59^{\mathrm{a}}$ & $4.16^{\mathrm{ab}}$ & $4.15^{\mathrm{a}}$ & $4.32^{\mathrm{a}}$ \\
$0 / 100$ & $4.62^{\mathrm{a}}$ & $4.52^{\mathrm{a}}$ & $3.82^{\mathrm{b}}$ & $4.07^{\mathrm{a}}$ \\
\hline Aedia/ asociado & $4.36^{\mathrm{a}}$ & $4.39^{\mathrm{a}}$ & $3.52^{\mathrm{bc}}$ & \\
\hline A,B Medias seguidas por letras mayúsculas diferentes en una misma columna difieren entre sí, \\
por la prueba de Tukey a 5\% de probabilidad. \\
a,b Medias seguidas por letras minúsculas diferentes en una misma fila difieren entre sí, por la \\
prueba de Tukey a 5\% de probabilidad
\end{tabular}


Cuadro 8. Efecto de la interacción Proporción x Asociado sobre el contenido de materia orgánica $(\% \mathrm{MO})$ de los asociados Avena/Vicia (A/V), Cebada/Vicia (C/V) y Trigo/Vicia (T/V) como cultivos hidropónicos

\begin{tabular}{ccccc}
\hline \multirow{2}{*}{ Proporciones } & \multicolumn{3}{c}{ Asociado } & $\begin{array}{c}\text { Media / } \\
\text { Proporción }\end{array}$ \\
\cline { 2 - 4 } & $\mathrm{A} / \mathrm{V}$ & $\mathrm{C} / \mathrm{V}$ & $\mathrm{T} / \mathrm{V}$ & $96.44^{\mathrm{a}}$ \\
\hline $100 / 0$ & $96.83^{\mathrm{a}}$ & $95.86^{\mathrm{ab}}$ & $96.62^{\mathrm{a}}$ & $96.29^{\mathrm{a}}$ \\
$60 / 20$ & $96.51^{\mathrm{a}}$ & $95.65^{\mathrm{b}}$ & $96.71^{\mathrm{a}}$ & $96.43^{\mathrm{a}}$ \\
$40 / 60$ & $96.66^{\mathrm{a}}$ & $96.41^{\mathrm{a}}$ & $96.23^{\mathrm{b}}$ & $95.70^{\mathrm{b}}$ \\
$20 / 80$ & $95.41^{\mathrm{b}}$ & $95.84^{\mathrm{ab}}$ & $95.85^{\mathrm{c}}$ & $95.69^{\mathrm{b}}$ \\
$0 / 100$ & $95.39^{\mathrm{b}}$ & $95.45^{\mathrm{b}}$ & $96.19^{\mathrm{b}}$ & $95.93^{\mathrm{b}}$ \\
\hline Media/ asociado & $95.69^{\mathrm{b}}$ & $95.62^{\mathrm{b}}$ & $96.48^{\mathrm{ab}}$ & \\
\hline A,B,C Medias seguidas por letras mayúsculas diferentes en una misma columna difieren entre \\
sí, por la prueba de Tukey a 5\% de probabilidad. \\
a,b,c Medias seguidas por letras minúsculas diferentes en una misma fila difieren entre sí, por \\
la prueba de Tukey a 5\% de probabilidad
\end{tabular}

\section{Minerales Totales (\%MM)}

El efecto del tipo de asociado, proporción e interacción presentó diferencias significativas ( $<<0.01$ ). La media de $4.62 \%$ (Cuadro 7) de materia mineral de la avena/vicia (A/V) en la proporción 20/80 fue significativamente mayor que el $3.18 \%$ de minerales totales de la proporción 100/0 (avena/vicia), asimismo, la $\mathrm{MM}$ de las proporciones de A/V 100/0, 80/20 y 60/40 fueron estadísticamente inferiores a las proporciones de 40/60, 20/80 y 0/100. Fuentes et al. (2011), por otra parte, reportó valores entre 4.59 y $5.57 \%$ en diferentes tiempos de cosecha para la avena, mientras que Carrasco y Espinoza (2016) encontraron un valor de $3.74 \%$.

El porcentaje de minerales totales de la mezcla C/V (100/0) de 4.14\% está en línea con el $4.6 \%$ obtenido por Carrasco y Espinoza (2016) para el cultivo hidropónico de cebada. Asimismo, el 3.38\% de minerales totales ob- tenido para el trigo (100/0) fue similar al 3.98\% reportado por Carrasco y Espinoza (2016), en condiciones de siembra semejantes. No obstante, Sánchez et al. (2013) reportaron valores superiores (5.80\% para zona de raíz y $5 \%$ para la parte aérea).

\section{Materia Orgánica (\%MO)}

El efecto del tipo de asociado, proporción e interacción presentó diferencias significativas $(\mathrm{p}<0.01)$. Las medias (Cuadro 8) muestran diferencias significativas entre todas las mezclas, siendo el mayor valor para trigo/vicia (96.35\%), seguida por avena/vicia. El efecto en la proporción se debe principalmente al asociado avena/vicia, donde el valor de la proporción 100/0 fue de 96.83, similar al porcentaje de MO obtenido por Carrasco y Espinoza (2016). Por otro lado, Contreras et al. (2015) reportaron 89.13\% de materia orgánica para la asociación cebada/vicia, siendo de $95.81 \%$ en el presente estudio. 


\section{Conclusiones}

- La producción de biomasa en las asociaciones cebada/vicia y trigo/vicia fue significativamente mayor que el asociado avena/vicia.

- El asociado avena/vicia tuvo el mayor porcentaje de materia seca.

- El porcentaje de proteína se incrementa entre la proporción $100 / 0$ a la proporción $0 / 100$ en todas las asociaciones.

- El asociado cebada/vicia tiene mayor porcentaje de FDN en su composición química en comparación las demás asociaciones.

- El mayor porcentaje de grasa se encontró en la proporción 100/0 en todas las asociaciones.

- $\quad \mathrm{E}$ l asociado $\mathrm{C} / \mathrm{V}$ presentó en promedio el menor porcentaje de materia orgánica.

\section{Literatura Citada}

1. AOAC. 1995. The official methods of analysis of AOAC International. $15^{\text {th }} \mathrm{ed}$. Washington: AOAC. $500 \mathrm{p}$.

2. Boue ST, Wiese S, Nehls M, Burow S, Elliott C, Carterwientjes B, Shih J, et al. 2003. Evaluation of the estrogenic effects of legume extracts containing phytoestrogens. J Agric Food Chem 51: 2193-2199.

3. Carrasco D, Espinoza D. 2016. Efecto de los cereales forrajes hidropónicos y los diferentes tiempos de cosecha sobre la composición química bromatológica y parámetros productivos. Tesis de Ingeniero Zootecnista. Huancavelica: Univ. Nacional de Huancavelica. 73 p.

4. Castro, J, Chirinos, D, Pineda M. 1998. Uso de forraje hidropónico de avena, cebada, y arveja en cuyes. En: XXI Reunión de la Asociación Peruana de Producción Animal. Puno, Perú: Univ. Nacional del Altiplano.
5. Cayllahua F, Condori DU, Cordero FA, veliz M, Contreras PJ. 2015. Sustitución gradual de la alfalfa (Medicago sativa $\mathrm{L}$ ) por el germinado de cebada (Hordeum vulgare) en raciones de cuyes (Cavia porcellus L) en la etapa de crecimiento. Rev Complutense Cienc Vet 9: 07-21.

6. Cerrillo M, Juárez A, Rivera J, Guerrero M, Ramírez, R, Bernal H. 2012 Producción de biomasa y valor nutricional del forraje verde hidropónico de trigo y avena. Interciencia 37: 906-913.

7. Chung TY, Nwokolo EN, Sim JS. 1989. Compositional and digestibility changes in sprouted barley and canola seeds. Plant Foods Hum Nutr 39: $267-$ 278. doi: 10.1007/BF01091937

8. Contreras P, José Luis, Tunque Q, Miguel, Cordero F, Alfonso G 2015. Rendimiento hidropónico de la arveja con cebada y trigo en la producción de germinados. Rev Inv Vet Perú 26: 9-19. doi: 10.15381/rivep.v26i1.10910

9. [FAO] Organización de las Naciones Unidas para la Alimentación y la Agricultura. 2001. Manual técnico: forraje verde hidropónico: Santiago de Chile: Oficina Regional de la FAO para América Latina y el Caribe. $68 \mathrm{p}$.

10. Fazaeli H, Golmohammadi HA, Shoayee AA, Montajebi N, Mosharraf Sh. 2011. Performance of feedlot calves fed hydroponics fodder barley. J Agr Sci Tech 13: 367-375.

11. Fuentes F, Poblete C, Huerta M, Palape I. 2011. Evaluación de la producción y calidad nutritiva de avena como forraje verde hidropónico en condiciones de desierto. Idesia 29: 75-81.

12. Ghazi, N, Karaki A, Hashimi M. 2012. Green fodder production and water use efficiency of some forage crops under hydroponic conditions. ISRN Agronomy ID 924672. doi:10.5402/2012/ 924672 
13. Guillermo J, Carpio H, Salvatierra J. 2006. La cuyecultura buscando ganancias esconómicas. En: VII Congreso Latinoamericano de Cuyecultura. Riobamba, Ecuador.

14. Jensen H, Malter A. 1995. Protected agriculture a global review. World Bank Technical Paper $\mathrm{N}^{\circ} 2.53 .156$ p.

15. Lomeli ZHM. 2000. Forraje verde hidropónico. El forraje del futuro. Hoy. Agrocultura 63: 15-18.

16. López PP, Guzmán OF, Santos LE, Prieto GF, Román GA. 2005. Evaluación de la calidad física de diferentes variedades de cebada (Hordeum sativum jess) cultivadas en el estado de Hidalgo y Talaxcala, México. Rev Chil Nutr 32: 247-253. doi: 10.4067/S071775182005000300010

17. López AR, Murillo AB, Rodríguez, QG. 2009. El forraje verde hidropónico $(\mathrm{FVH})$ : una alternativa de producción de alimento para el ganado en zonas áridas. Interciencia 34: 121-126.

18. Mazuelos CV. 1995. Utilización de la cebada (Hordeum vulgare) y maíz (Zea mays) germinados en la alimentación de cuyes hembras durante el empadre, gestación y lactancia. Tesis de Ingeniero Zootecnista. Lima, Perú: Univ. Nacional Agraria La Molina. $95 \mathrm{p}$.

19. Mooney J. 2005. Growing cattle feed hydroponically. Meat and Livestock Australia. $30 \mathrm{p}$.
20. Núñez TO, Guerrero LJ. 2021. Forrajes hidropónicos: una alternativa para la alimentación de animales domésticos. J Selva Andina Anim Sci 8: 44-52.

21. Pacco JC. 2018. Producción de forraje verde hidropónico de cebada y avena con adición de fitohormonas en Cabana - Puno. Tesis de ingeniero agrónomo. Puno, Perú: Universidad Nacional del Altiplano. $122 \mathrm{p}$.

22. Romero ME, Córdova G, Hernández EO. 2009. Producción de forraje verde hidropónico y su aceptación en ganado lechero. Acta Universitaria 19: 11-19.

23. Sánchez F, Moreno EC, Contreras E, Morales J. 2013. Producción de forraje hidropónico de trigo y cebada y su efecto en la ganancia de peso en borregos. Rev Chapingo Ser Hortic 19(4): 35-43.

24. Silva E. 1994. Utilización de la cebada (Hordeum vulgare) y maíz (Zea mays) germinado en la alimentación de cuyes machos en crecimiento y engorde. Tesis de Ingeniero Zootecnista. Lima, Perú: Univ. Nacional Agraria La Molina. $110 \mathrm{p}$.

25. Sneath R, Macintosh F. 2003. Review of hydroponic fodder production for beef cattle. Meat Livestock Aust. 54 p.

26. Villanueva J, San Martin F. 1997. Alimentación de vaquillas en crecimiento a base de residuos de cosecha tratada con urea y suplementadas con proteína sobrepasante. Rev Inv Pec IVITA 8(1): $39-48$. 